\title{
Routine Contrast Esophagram in Anastomotic Leaks After Esophagectomy; Methodological Issues on Diagnostic Value: Reply
}

\author{
Zhengbin You ${ }^{1}$
}

Published online: 10 May 2017

(c) Société Internationale de Chirurgie 2017

\section{Response:}

First, we would like to thank Dr. Sabour for his letter regarding our report [1]. Diagnostic value must be considered as a combination of both diagnostic accuracy (validity) and diagnostic precision (reliability). Although some appropriate tests relevant to diagnostic accuracy were mentioned in our paper, diagnostic precision (reliability) should be assessed as a unique methodological issue of diagnostic value. Receiver operating characteristic (ROC) curve analysis should be used in clinical studies to assess diagnostic value. In our paper, the esophagram sensitivity was $93.3 \%$ in the intrathoracic group, which is significantly greater than the value of $76.2 \%$ mentioned in Sabour's letter, which was based on the ratio of true positive cases $(n=16)$ to all intrathoracic leaks $(n=21)$. Among the relevant studies cited in our paper [2-4], none calculated ROC curves. In our paper, the ROC was lower in the cervical group than in the intrathoracic group [0.682 (range, 0.5-0.7) vs. 0.967 (range, 0.9-1), respectively]. For an esophagram of the neck, the sensitivity was $38.9 \%$ and the specificity was $97.6 \%$. The aim of our study was to exclude leaks before oral eating using routine contrast esophagram. Although the specificity was higher, this modality had no value to preclude leaks in the cervical group. Although the ROC was 0.682 , the precision was relatively low. Since 2015 , the integrity of a cervical anastomosis in our center is tested by drinking small amounts of water with simultaneous observation of the cervical wound without the use of routine contrast esophagram. For the intrathoracic group, the sensitivity and specificity were relatively high, as was the ROC curve, which was almost 1.

Thank you again for your letter. In the future, we will pay more attention to diagnostic precision and accordingly present our data more comprehensively and effectively.

\section{References}

1. Hu Z, Wang X, An X et al (2017) The diagnostic value of routine contrast esophagram in anastomotic leaks after esophagectomy. World J Surg. doi:10.1007/s00268-017-3923-9

2. Tirnaksiz MB, Tirnaksiz MB (2005) Effectiveness of screening aqueous contrast swallow in detecting clinically significant anastomotic leaks after esophagectomy. Eur Surg Res 37:123-128

3. Solomon DG, Sasaki CT, Salem RR (2012) An evaluation of the routine use of contrast radiography as a screening test for cervical anastomotic integrity after esophagectomy. Am J Surg 203(4): 467-471

4. Boone J, Rinkes IB, van Leeuwen M, van Hillegersberg R (2008) Diagnostic value of routine aqueous contrast swallow examination after oesophagectomy for detecting leakage of the cervical oesophagogastric anastomosis. ANZ J Surg 78(9):784-790
Zhengbin You

yhayyxw@163.com

1 Division of Thoracic Surgery, Huai'an First People's Hospital, Nanjin Medical University, Huai'an, Jiangsu, China 